\title{
Inappropriate use of proton pump inhibitors in a local setting
}

\author{
Christopher Tze Wei Chia ${ }^{1}$, MBBS, MRCP, Wan Peng $\underline{\operatorname{Lim}}^{1}$, BSc, Charles Kien Fong Vu${ }^{1}$, MBBS, FRACP
}

INTRODUCTION There are growing concerns that the use of proton pump inhibitors (PPIs) may be inappropriate in instances that do not conform to evidence-based indications. This point-prevalence study aimed to investigate the frequency, indications and appropriateness of use of PPIs in hospitalised patients on a randomly chosen day.

METHODS On a randomly chosen day, all inpatients were documented, and those on any form of PPIs on that day were determined. Indications for maintaining these patients on PPIs were obtained from the electronic medical records, which were then recorded and cross-referenced against a list of accepted indications adapted from the US Food and Drug Administration (FDA)-approved list.

RESULTS In all, 1,025 inpatients were documented. Of the 477 (46.5\%) inpatients using PPIs, only 219 (45.9\%) fulfilled the FDA-approved indications, while the majority $(n=258,54.1 \%)$ did not. Overall, PPIs were not strictly indicated for use in 206 (43.2\%) inpatients, according to FDA criteria. Of the 477 inpatients on PPIs, 52 (10.9\%) had borderline indications based on expert consensus/guidelines other than FDA criteria.

CONCLUSION Although the use of PPIs is prevalent in hospitals, less than half of the hospitalised patients using PPIs in our study had evidence-based indications that supported such use. The overuse of PPIs has a negative impact on healthcare costs and may lead to adverse effects. Steps to curb the inappropriate use of PPIs should address factors such as indications for the initiation of PPIs, and reassessment of the need for ongoing PPI use in inpatients upon discharge and during outpatient reviews.

Keywords: guidelines, hospitalised, inappropriate use, patients, proton pump inhibitors

\section{INTRODUCTION}

Polypharmacy is becoming more prevalent with advances in the pharmaceutical sciences and the development of newer, though not necessarily better, drugs. One class of medications that has enjoyed steady popularity is proton pump inhibitors (PPIs), which appears near the top of many lists of the most commonly prescribed medications. ${ }^{(1)}$ However, studies show that a significant number of PPI prescriptions among both inpatient and outpatient populations is often inappropriate and adds to additional healthcare costs while contributing to long-term adverse effects. ${ }^{(2)}$ For instance, a longitudinal study from the United States showed that $69 \%$ of PPI prescriptions lacked appropriate medical indications, contributing to a total PPI drug cost of USD 3,013,069 during the first 30 days post-discharge. ${ }^{(3)}$ Similarly, another study that compared PPI usage in academic and nonacademic hospitals showed that only $39 \%$ of PPI indications were guideline-compliant. ${ }^{(4)}$ Additionally, a recent study from two academic hospitals in Lima, Peru, reported an almost $54 \%$ incidence of inappropriate PPI use. ${ }^{(5)}$

Although non-evidence-based indications are commonplace, not much data is available on the inappropriate use of PPIs in Asia. Given the paucity of such data in the literature, a pointprevalence study was conducted with the aim of identifying the prevalence, indications and appropriateness of PPI use in a local tertiary hospital setting in Singapore.

\section{METHODS}

This point-prevalence study was conducted on a randomly chosen day after we had analysed the hospital ward census records for the total number of inpatients seen from June to July 2011 across all disciplines in Tan Tock Seng Hospital, Singapore. This was done to ensure that no significant fluctuations were observed on any particular day and that the inpatient numbers on the chosen day did not fluctuate greatly from the daily average at the hospital. The total number of inpatients on the chosen day and inpatients who were on PPIs were identified via the hospital electronic medical records, which also included details pertaining to the patients' presenting complaints, duration of symptoms, salient features of the history of presenting complaints, social history, past medical history, family history, records of previous admissions, physical examination findings, investigations performed, summary of management instituted and records of previous medications prescribed by the study hospital and its affiliates (i.e. other government/restructured hospitals and healthcare facilities, excluding private institutions and general practitioners).

One of the advantages of using the electronic medical records at our hospital was that all patients' previously prescribed medications and medical records from other affiliated institutions could be viewed and their indications for PPI use reviewed based on definite documentation, suggestive symptoms or indications based on clinical circumstances. For patients already on PPIs before admission, the electronic medical records allowed for a

${ }^{1}$ Department of Gastroenterology and Hepatology, Tan Tock Seng Hospital, Singapore

Correspondence: Dr Christopher Chia Tze Wei, Registrar, Department of Gastroenterology and Hepatology, Tan Tock Seng Hospital, 11 Jalan Tan Tock Seng, Singapore 308433. christopher_tw_chia@ttsh.com.sg 
search of previous prescription records to identify possible/definite indications for PPI use.

Apart from the hospital electronic medical records, indications for PPI use were also sourced from the discharge summaries of these patients. These indications were then crossreferenced with those approved by the United States Food and Drug Administration (FDA), as shown in Table I. ${ }^{(6)}$ For patients in whom indications to start or continue PPI were unclear from the electronic medical records, the actual case notes on admission were reviewed to determine the possible reasons for PPI initiation or continuation, where applicable.

Inpatients on PPIs were categorised into three groups, according to: (a) those who fulfilled the FDA indications; (b) those who had no clear indications; and (c) those with borderline indications. 'Borderline indications' was defined as indications that were not strictly FDA-approved but deemed acceptable on the basis of general expert consensus, or based on guidelines other than the FDA, such as those from the UK National Institute for Health and Clinical Excellence (NICE)(7) and American College of Gastroenterology (ACG). ${ }^{(8)}$ Table II shows other accepted or off-label indications for PPI use. ${ }^{(7,8)}$

As this was a retrospective survey, no blinding was necessary. This survey was initially conducted as part of the hospital medical board's initiative to gauge the prevalence of PPI use in our centre and whether indications were appropriate among patients on PPIs. No approval was required from the National Health Group Domain Specific Review Board.

\section{RESULTS}

Among the 1,025 inpatients seen on the chosen day, 477 (46.5\%) inpatients were on PPIs (Fig. 1). Of these 477 patients, 219 (45.9\%) fulfilled the FDA-approved indications, 206 (43.2\%) had no clear indications for PPI use (Table III), and 52 (10.9\%) had borderline indications based on expert consensus/guidelines other than the FDA criteria (Table IV).

The electronic medical records and discharge summaries of 47 inpatients did not suggest any possible reason for PPI use. For these patients, we traced and manually reviewed the actual case notes to ensure that there were no hidden indications for PPI use that were not reflected in their electronic medical records or discharge summaries. Among patients on PPIs with no clear indications, anaemia (with no other concomitant symptoms such as asymptomatic anaemia, based on documentation in the electronic records) was the main non-indication associated with inappropriate PPI use. Other subsequent reasons behind inappropriate PPI use besides anaemia were found to be as follows: (a) the use of aspirin/nonsteroidal anti-inflammatory drugs (NSAIDs) in patients aged $<65$ years without other risk factors for gastrointestinal tract bleeding (21.8\%); (b) no documented indications found in the electronic medical records (11.7\%); (c) use of corticosteroids (7.3\%); (d) warfarin $(6.8 \%)$; (e) malignancy $(6.8 \%)$; (f) skeletal fractures $(2.4 \%)$; and (g) other isolated indications that did not fulfil the FDA criteria $(8.7 \%)$.

Borderline indications seen in our study included, in descending order of frequency, endoscopic findings of gastric
Table I. United States Food and Drug Administration accepted indications for the use of proton pump inhibitors. ${ }^{(6)}$

\begin{tabular}{ll}
\hline Indication & Approved use \\
\hline Peptic ulcer disease & $\begin{array}{l}\text { Treatment of duodenal } \\
\text { ulcer/gastric ulcer }\end{array}$ \\
Erosive oesophagitis & Healing and maintenance \\
Helicobacter pylori & $\begin{array}{l}\text { Eradication with appropriate } \\
\text { antibiotic regimen }\end{array}$ \\
Gastro-oesophageal reflux disease & Symptomatic treatment \\
Pathological hypersecretory & Zollinger-Ellison syndrome \\
conditions & In critically ill patients \\
Stress ulcer prophylaxis & \\
\hline
\end{tabular}

Table II. Other accepted/off-label indications for the use of proton pump inhibitors.*

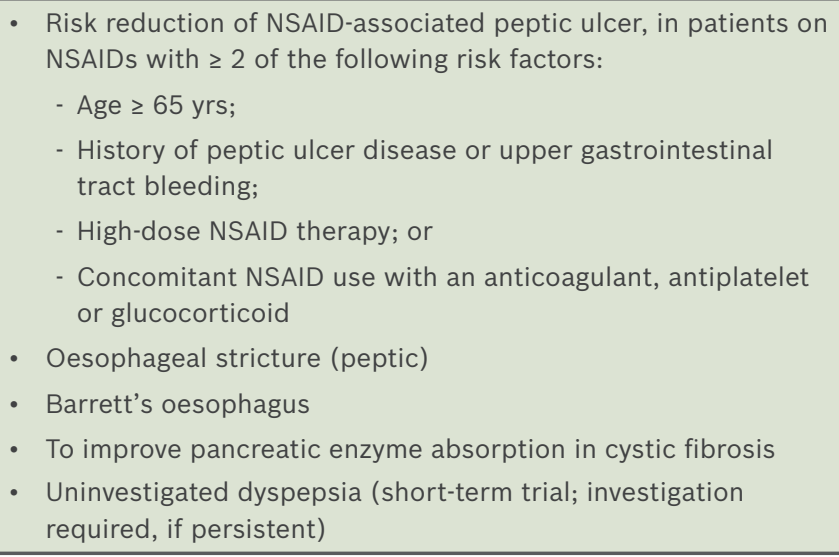

*According to other peer-reviewed clinical studies/guidelines such as those from the National Institute for Health and Clinical Excellence ${ }^{(7)}$ and the American College of Gastroenterology. ${ }^{\left({ }^{(8)}\right.}$ NSAIDs: nonsteroidal anti-inflammatory drugs

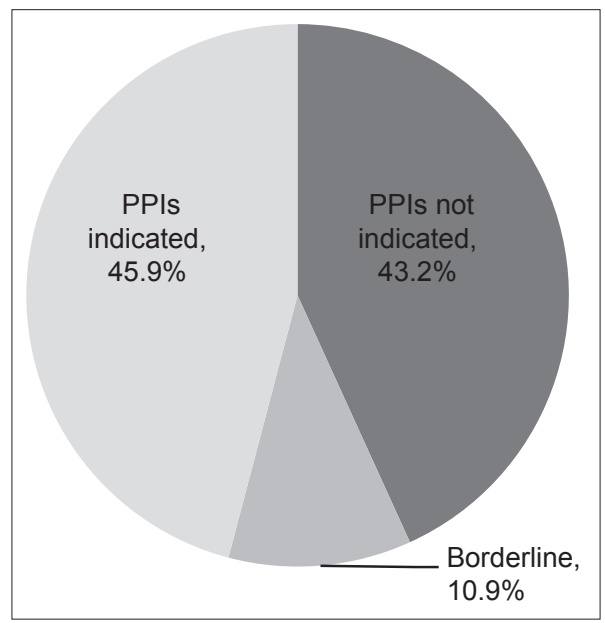

Fig. 1 Distribution of inpatients on proton pump inhibitors (PPIs) ( $n=477)$.

erosions/pangastritis/gastritis (34.6\%), patients on dual antiplatelet agents $(15.4 \%)$, anaemic patients who were unstable or had a history of gastrointestinal tract bleeding $(13.5 \%)$, patients with uninvestigated dyspepsia (7.7\%), patients on dual antiplatelet agents with concurrent anaemia $(7.7 \%)$, and other miscellaneous borderline/soft indications (e.g. recent step-down care from the high-dependency ward or steroid use in advanced malignancy) $(21.1 \%)$ 


\begin{tabular}{|c|c|}
\hline Non-indication & No. (\%) \\
\hline $\begin{array}{l}\text { Anaemia (no evidence of gastrointestinal tract } \\
\text { bleeding/clinically stable) }\end{array}$ & $71(34.5)$ \\
\hline Aspirin, antiplatelet or NSAIDs (age $<65$ yrs) & $45(21.8)$ \\
\hline No apparent indication (as shown in the EMR) & $24(11.7)$ \\
\hline Corticosteroids & $15(7.3)$ \\
\hline Warfarin & $14(6.8)$ \\
\hline Malignancy & $14(6.8)$ \\
\hline Bone fracture & $5(2.4)$ \\
\hline Other* & $18(8.7)$ \\
\hline
\end{tabular}

*This includes nasogastric tube feeding, nil per os status, on low-molecular-weight heparin, musculoskeletal chest pain, bone fracture, foreign body removal in throat, high gastric residual volume and post-tonsillectomy. EMR: electronic medical record; NSAIDs: nonsteroidal anti-inflammatory drugs

Table IV. Patients with borderline indications for the use of proton pump inhibitors $(n=52)$.

\begin{tabular}{lc}
\hline Borderline indication* $^{*}$ & No. (\%) \\
\hline Endoscopy (pangastritis/erosions) & $18(34.6)$ \\
Double antiplatelet agents & $8(15.4)$ \\
Anaemia (high-risk, clinically unstable/with & $7(13.5)$ \\
possible history of gastrointestinal bleeding) & \\
Double antiplatelet + anaemia & $4(7.7)$ \\
Uninvestigated dyspepsia & $4(7.7)$ \\
Other $^{+}$ & $11(21.1)$ \\
\hline
\end{tabular}

*Borderline indications include indications based on general consensus or other non-FDA guidelines such as those from the National Institute of Clinical Excellence and the American College of Gastroenterology, or indications that are deemed

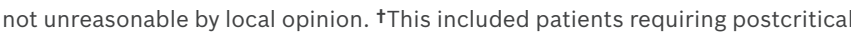
care, such as recent step-down care from intensive care unit/coronary care unit, or those on steroids due to advanced malignancy.

\section{DISCUSSION}

Our study shows that less than $50 \%$ of PPIs prescribed to our inpatients were indicated based on FDA-approved criteria and that the main inappropriate indication was simple anaemia. Even after extending the definition of indications for PPI use to include those espoused by general expert consensus and in other guidelines such as NICE and ACG, nearly half of all PPI prescriptions $(43.2 \%)$ at our centre were found to have no clear indication for their initiation or continued use. This finding was in accordance with previous Western studies, which supported the notion that PPIs are one of the most overprescribed medicines in the medical world..$^{(1,2,9-14)}$ For instance, a PubMed literature search on 'proton pump inhibitor overuse' showed that the ballpark figure for inappropriate PPI use ranged from $40 \%$ to $81 \%$, with a mean of $63 \% .^{(4,5,9,10,12-21)}$ Such widespread and inappropriate use of PPIs is likely to translate into possible significant long-term adverse effects such as increased gastrointestinal tract infections (e.g. Clostridium difficileassociated diarrhoea), pneumonia and fracture risks. ${ }^{(22-28)}$ Undoubtedly, healthcare costs and the accompanying financial burden would escalate due to the inappropriate initiation and maintenance of this large group of patients on PPIs, should no reconciliatory measures be taken.
There were several limitations to our study, including the retrospective nature of the survey. As only a single tertiary centre was surveyed, it may not be possible to generalise our results to the entire Asian population - the inclusion of other multiregional centres may have better represented the prevalence of PPI use in Asia or Southeast Asia. Nevertheless, we are of the opinion that the overall conclusions of a more extensive Asian study are unlikely to significantly differ from our findings, as our results were consistent with previous studies in Western populations. Our study duration was short, with the survey based on a oneday analysis of all patients in our hospital, although efforts were made to ensure that the total number of inpatients and those on PPIs were within the average range for most days. It is also possible that the documentation of indications for PPI use in some electronic medical records was not comprehensive enough in our study. Insufficient documentation could have resulted in an underestimation of the number of patients with actual indications by categorising them as non-indicated patients instead. Efforts were undertaken to manually review the case notes of patients who had no indications for PPI use in their electronic medical records and discharge summaries. However, for many such patients, the case notes had no mention or documentation of the reasons for the initiation of PPIs or the continuation of previously prescribed PPIs. Thus, for most patients not indicated for PPI use in the electronic medical records and discharge summaries, manual review of case notes did not add much value, especially where the problem lists, investigations and management were well summarised in the electronic records. We also did not investigate PPI indications in patients with outpatient prescriptions for PPIs. It would be interesting to determine whether hospitalist and non-hospitalist practices differ significantly when it comes to PPI prescription. For instance, a Spanish study on a hospital cohort found that hospitalisation did not represent an opportunity for better prescription of PPIs. ${ }^{(9)}$

Given the magnitude of the problem of inappropriate PPI prescription in medical practice, remedial measures to improve the situation should be considered with alacrity. One possible approach would be to educate medical departments and teams most associated with inappropriate PPI prescriptions in hospitals, as well as junior doctors who most often initiate PPI use in the wards. Another practical strategy would involve having dedicated pharmacists in the wards or during ward rounds, who could monitor PPI use and undertake medical reconciliation during the admission, step-down care (from high-dependency units to general wards) and discharge processes. This could help to limit the prescription of PPIs to patients with more appropriate indications, as the pharmacists could suggest the discontinuation of PPIs in patients when it is no longer indicated to ward doctors. In our tertiary hospital, where almost all prescriptions are initiated electronically, a computer-based or online stewardship guide developed for clinicians, which shows the appropriateness of indications for PPI use each time PPIs are ordered online/ electronically, could greatly influence and restrict the prescription of these drugs to patients with more appropriate indications. When extrapolated to the outpatient clinical setting, the institution 
of such a system could control the rampant misuse of PPIs in outpatient clinics.

In summary, the present study concludes that PPI use is prevalent in Singapore hospitals and approximately half of the overall usage may not be clearly indicated. To our knowledge, this is one of the first Asian studies to demonstrate that the overuse

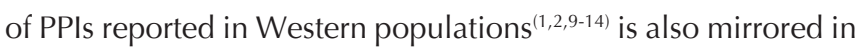
Asian populations. In view of the universality of inappropriate PPI prescription in medical practice, the drafting and institution of urgent measures to address this troubling problem is necessary.

\section{ACKNOWLEDGEMENTS}

We would like to acknowledge the invaluable input received from colleagues in the Department of Gastroenterology and Hepatology, Tan Tock Seng Hospital (TTSH), Singapore, during the course of this study, and Ms Hoo Si Ru, Pharmacist, at the Department of Pharmacy, TTSH, for her assistance during data acquisition.

\section{REFERENCES}

1. Regal RE, Osta AD, Parekh VI. Interventions to curb the overuse of Acidsuppressive medications on an inpatient general medicine service. P T 2010; 35:86-90.

2. Heidelbaugh JJ, Goldberg KL, Inadomi JM. Magnitude and economic effect of overuse of antisecretory therapy in the ambulatory care setting. Am J Manag Care 2010; 16:e228-34.

3. Thomas L, Culley EJ, Gladowski P, et al. Longitudinal analysis of the costs associated with inpatient initiation and subsequent outpatient continuation of proton pump inhibitor therapy for stress ulcer prophylaxis in a large managed care organization. J Manag Care Pharm 2010; 16:122-9.

4. Eid SM, Boueiz A, Paranji S, et al. Patterns and predictors of proton pump inhibitor overuse among academic and non-academic hospitalists. Intern Med 2010; 49:2561-8.

5. Bustamante Robles KY1, Ticse Aguirre R, Cánepa Rondo IF, et al. [Frequency of proton pump inhibitor prescription based in clinical practice guidelines in hospitalized patients in two academic hospitals in Lima, Peru]. Rev Gastroenterol Peru 2012; 32:44-9. Spanish.

6. FDA Center for Drug Evaluation and Research. New Drug Application (NDA) Nexium (esomeprazole magnesium) delayed release capsules. AstraZeneca LP. Application Number 21-153 \& 21-154 [online]. Available at: http://www.accessdata.fda.gov/drugsatfda_docs/ nda/2004/021153Orig1s008.pdf. Accessed March 11, 2013.

7. National Institute for Clinical Excellence. Guidence of the use of proton pump inhibitors in the treatment of dyspepsia [online]. Available at: www. nice.org.uk/nicemedia/pdf/proton.pdf. Accessed March 11, 2013.

8. Lanza FF, Chan FK, Quigley EM, Practice Parameters Committee of the American College of Gastroenterology. Guidelines for prevention of NSAID-related ulcer complications. Am J Gastroenterol 2009; 104:728-38.
9. Ramirez E, Lei SH, Borobia AM, et al. Overuse of PPIs in patients at admission, during treatment, and at discharge in a tertiary Spanish hospital. Curr Clin Pharmacol 2010; 5:288-97.

10. Nardino RJ, Vender RJ, Herbert PN. Overuse of acid-suppressive therapy in hospitalized patients. Am J Gastroenterol 2000; 95:3118-22.

11. Salmasian H, Freedberg DE, Abrams JA, Friedman C. An automated tool for detecting medication overuse based on the electronic health records. Pharmacoepidemiol Drug Saf 2013; 22:183-9.

12. Gupta R, Garg P, Kottoor R, et al. Overuse of acid suppression therapy in hospitalized patients. South Med J 2010; 103:207-11

13. Chavez-Tapia NC, Tellez-Avila FI, Garcia-Leiva J, Valdovinos MA. Use and overuse of proton pump inhibitors in cirrhotic patients. Med Sci Monit 2008; 14:CR468-72.

14. Van Vliet EP, Otten HJ, Rudolphus A, et al. Inappropriate prescription of proton pump inhibitors on two pulmonary medicine wards. Eur J Gastroenterol Hepatol 2008; 20:608-12.

15. Bez C, Perrottet N, Zingg T, et al. Stress ulcer prophylaxis in non-critically ill patients: a prospective evaluation of current practice in a general surgery department. J Eval Clin Pract 2012; 19:378-8.

16. Pasina L, Nobili A, Tettamanti M, et al. Prevalence and appropriateness of drug prescriptions for peptic ulcer and gastro-esophageal reflux disease in a cohort of hospitalized elderly. Eur J Intern Med 2011; 22:205-10.

17. Glew CM, Rentler RJ. Use of proton pump inhibitors and other acid suppressive medications in newly admitted nursing facility patients. J Am Med Dir Assoc 2007; 8:607-9.

18. Grube RR, May DB. Stress ulcer prophylaxis in hospitalized patients not in intensive care units. Am J Health Syst Pharm 2007; 64:1396-400.

19. Scagliarini R, Magnani E, Praticò A, et al. Inadequate use of acidsuppressive therapy in hospitalized patients and its implications for general practice. Dig Dis Sci 2005; 50:2307-11.

20. Strid H, Simrén M, Björnsson ES. Overuse of acid suppressant drugs in patients with chronic renal failure. Nephrol Dial Transplant 2003; 18:5705 .

21. Naunton M, Peterson GM, Bleasel MD. Overuse of proton pump inhibitors. J Clin Pharm Ther 2000; 25:333-40.

22. Omeprazole: Drug Information Lexicomp [online]. Available at: www. UpToDate.com. Accessed June 2011.

23. Eisai Inc. Aciphex prescribing information [online]. Available at: http://dailymed.nIm.nih.gov/dailymed/archives/fdaDrugInfo. cfm?archiveid=10184. Accessed March 11, 2013.

24. AstraZeneca LP. Nexium prescribing information [online]. Available at: http://www1.astrazeneca-us.com/pi/Nexium.pdf. Accessed March 11, 2013.

25. TAP Pharmaceuticals Inc. Prevacid prescribing information [online]. Available at: http://www.accessdata.fda.gov/drugsatfda_docs/ label/2008/020406s067,021281s024,021428s01 7lbl.pdf. Accessed March $11,2013$.

26. AstraZeneca LP. Prilosec prescribing information [online]. Available at: http://www1.astrazeneca-us.com/pi/Prilosec.pdf. Accessed March 11, 2013.

27. Wyeth Pharmaceuticals Inc. Protonix prescribing information [online]. Available at: http://labeling.pfizer.com/showlabeling.aspx?id=135. Accessed March 11, 2013.

28. Santarus Inc. Zegerid prescribing information [online]. Available at: http:// www.zegerid.com/docs/zegerid-full-prescribing-information.pdf. Accessed March 11, 2013. 